\title{
Design of Reflective Filters Based on Organic Materials Using Genetic Algorihtms
}

\author{
Y. K. Bai, Aleksandra B. Djurišić, Weiling Guo, and E. Herbert Li \\ ${ }^{a}$ Department of Electrical \& Electronic Engineering, University of Hong Kong, \\ Pokfulam Road, Hong Kong
}

\begin{abstract}
In this work we propose a genetic algorithm for the design of reflective filters consisting of organic materials. The algorithm chooses the materials of the layers from the list of available materials and chooses their thickness in order to obtain optimal reflectance characteristics. The wavelength dependence of the refractive index and the coefficient of extinction of the layers is taken into account.
\end{abstract}

Keywords: Genetic algorithms, Bragg reflectors

\section{INTRODUCTION}

Organic light emitting diodes (OLEDs) are being intensively investigated in terms of efficient materials and device structures for their great potential in the huge display market. In spite of the increasingly high internal quantum efficiency, the OLED external quantum efficiency is significantly reduced by total internal reflection inside the device. The output light outside the device can be increased via optical microcavity. With an OLED structure placed between two reflectors, the emission wavelength, which is resonant with the cavity modes, is enhanced in the emission spectrum, while the others are suppressed. Usually, one reflector is the top metal electrode ( $\mathrm{Al}$ or $\mathrm{Mg}: \mathrm{Ag}$ ) while the other can be a thin metal layer, or a dielectric stack. ${ }^{1}$ In the latter case, the hole injecting contact, usually indium tin oxide (ITO), is placed inside the cavity, which degrades its quality factor. In most cases, we prefer DBR mirror as the outlet of light because of its low absorption in contrast with metal mirrors.

In this work, we present a method for the design of a reflector made of organic small molecule materials which can be used to design an all-organic microcavity OLED. The method is based on a genetic algorithm. Genetic algorithms represent a global optimization technique which mimic the concept of natural evolution, by applying the evolution operators (selection, crossover, and mutation) to the set of possible solutions, called population. The population consists of vectors of variables, which are in this case pairs of material code (which determines which material from the list will be chosen) and layer thickness. The algorithm is highly versatile, and it enables design of asymmetric filter structures, which involve more than two materials, as well as the conventional two-material reflectors. The list of available materials presently includes N,N'diphenyl-N,N'-bis(3-methyl-phenyl)-1,1'biphenil-4,4'diamine (TPD), copper-phthalocyanine (CuPc) and N,N'dimethylperylene-3,4:9,10-dicarboximide. The list can be further extended with suitable organic materials, which do not absorb in the spectral region of interest. The optical properties of all the materials considered have been described with a modified Lorentz oscillator model, so that the wavelength dependence of both the refractive index and coefficient of extinction is fully taken into account.

The reflectance characteristics of the reflectors, which are designed for the structure glass/ indium-tin oxide (ITO)/reflector/TPD, is calculated by means of the genetic algorithm. In the structure ITO serves as a hole injecting contact, and TPD is a hole transport layer. The reflectors have been designed to have peak reflectivity in the blue-green spectral region. Tris-(8-hydroxyquinoline) aluminum (Alq3) represents the most commonly used luminescent material in OLEDs, $\mathrm{Alq}_{3}$ has emission maximum in green spectral region. The peak wavelength of the reflectance filter is chosen at low energy side of $\mathrm{Alq}_{3}$ emission spectrum due to unavailability of the index of refraction of organic materials transparent between $520 \mathrm{~nm}$ and $560 \mathrm{~nm}$.

•E_mail: ehli@eee.hku.hk; Fax: +852 2559 8738; Tel: +852 28597091 
Due to generality of the design method, the design objective can be easily changed by manipulating the objective function which is minimized by the genetic algorithm. In the design presented here, the reflectivity peak was chosen to be at $470 \mathrm{~nm}$ or $490 \mathrm{~nm}$, and the magnitude of the reflectivity peak has been determined from considerations of emission enhancement in a microcavity device. The enhancement of the emission intensity along the cavity axis at the resonance wavelength is given by $^{4}$

$$
G_{c a v}=\frac{\varsigma}{2} \frac{\left(1+\sqrt{R_{1}}\right)^{2}\left(1-R_{2}\right)}{\left(1-\sqrt{R_{1} R_{2}}\right)^{2}} \frac{\tau_{c a v}}{\tau},
$$

where $R_{1}$ is the reflectivity of the top metal mirror, $R_{2}$ is the reflectivity of the bottom mirror, $\zeta$ is the antinode enhancement factor, and $\tau_{c a v} \tau$ is the ratio of exciton lifetimes in the cavity device and non-cavity device. The factor depending on the reflectivities of the mirrors has a maximum in the region $0.8<R_{2}<0.95$ for $R_{1}>0.8$, whose exact position depends on the reflectance of the top metal mirror. The desired magnitude of reflectance peak should be chosen in such a manner that we obtain the maximum value of this factor for the defined reflectivity of the top metal mirror. We show that the reflectance characteristics close to the desired one can be obtained using both asymmetrical filters which are not limited to the choice of two materials, and more conventional two-material reflectors. Since all the layers in the reflector are organic semiconductors, use of such a reflector in OLED would enable placing the ITO electrode outside the cavity, which can significantly shorten the cavity length. Shorter cavity leads to a reduction in the angle and wavelength dependence of the emission. ${ }^{1}$ However, the true influence of such all-organic reflector to the carrier transport in OLEDs needs further investigation.

It is our main objective to demonstrate that the reflective filter consisting of organic layers can be designed to have a suitable reflectance characteristic for application in microcavity OLEDs operating in blue-green spectral region. The paper is organized as follows. In the following Section, we describe the genetic algorithm used in filter design. In Section III, results and discussion are presented. Finally, conclusions are drawn.

\section{DESCRIPTION OF THE ALGORITHM}

Elite genetic algorithm with adaptive mutations (EGAAM) ${ }^{5}$ is adopted in our work. Genetic algorithms represent global optimization technique which is based on the analogy with evolution. An illustration of a genetic algorithm is shown in Fig. 1. Genetic algorithm implementation requires defining the coding scheme, initial population generation, and selection, crossover and mutation operators. Floating-point coding scheme $\mathrm{e}^{5-7}$ has been employed. Each layer is characterized with two real numbers, namely alloy composition and layer thickness. In this coding scheme, each gene has the value of the corresponding variable $p(k), k=1, n_{v}$, where $n_{v}$ is the number of variables. The number of variables differs for three design procedures. In the design procedure c), composition and thickness of each layer are chosen independently, and the total number of variables is equal $2 \times n_{l}$, where $n_{l}$ is the number of layers. In design procedure b), two materials are used while layer thickness of each layer is determined independently, and the number of variables is $n_{l}+2$, for two layer compositions and $n_{l}$ layer thickness parameters. In design procedure a), the algorithm chooses only alloy composition and layer thickness for two layers, which are repeated designated times, so that the number of variables is 4 regardless of the number of layers. Among the above procedures, design procedure a) is the closest to the conventional quarter-wave design. Use of genetic algorithm enables us to find an optimal design within given constraints, which represents an advantage over the conventional design. According to the following equation ${ }^{17}$ the values of variables in the initial population are generated in all cases

$$
p(k)=p_{l}(k)+\left(p_{u}(k)-p_{l}(k)\right) \times r \quad,
$$

where $r$ is a random number $r \in[0,1]$, and $p_{l}(k)$ and $p_{u}(k)$ are initially set lower and upper boundary, respectively. Restricting the variables in the specified domain is achieved in the above method, which insures that variables have physical meaning, i.e. that all the thickness values are positive and all the compositions $0 \leq x \leq 1$. 


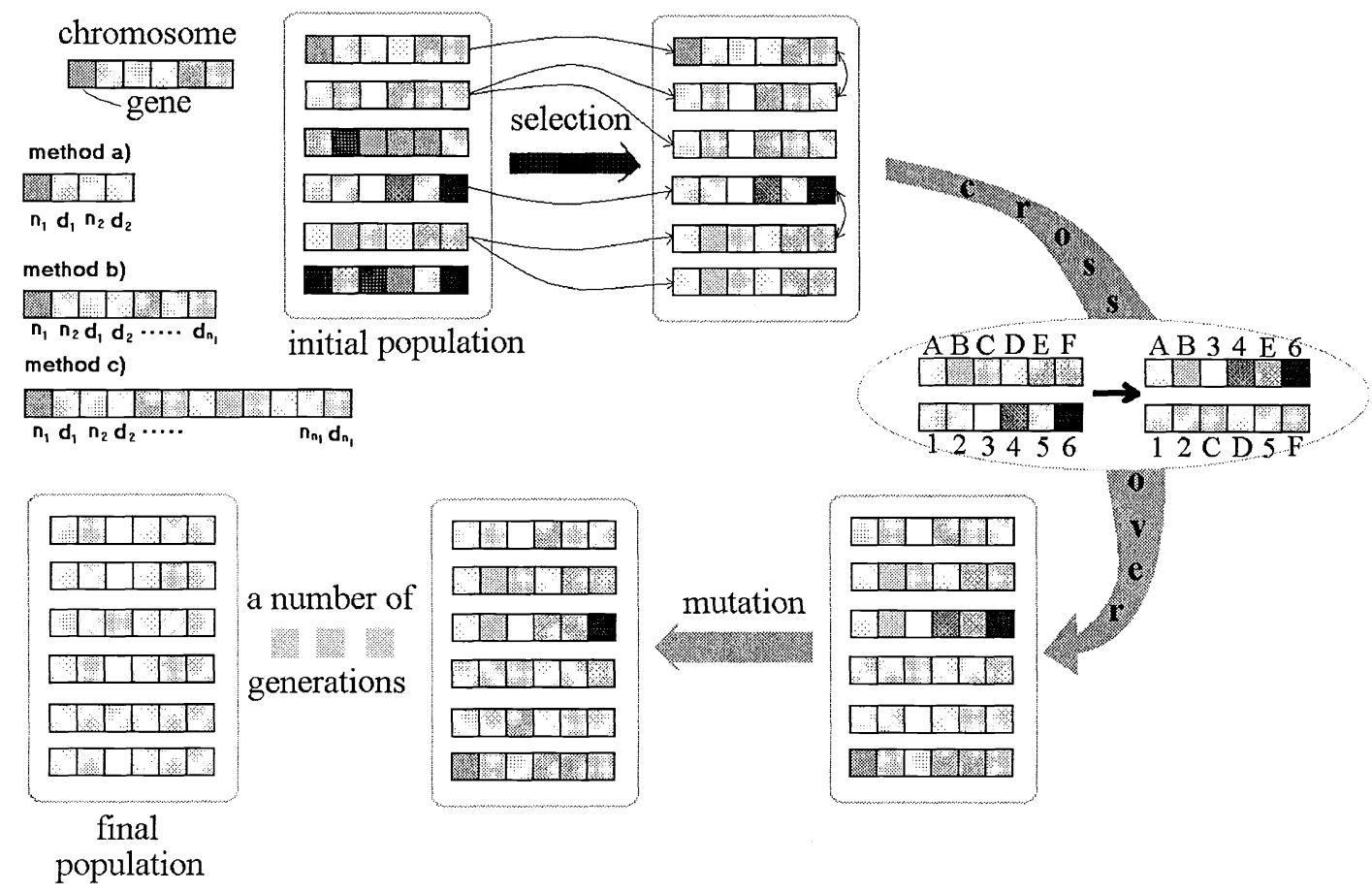

Fig. 1 Illustration of a genetic algorithm. Shade of gray indicates fitness: lighter shade, better fitness.

EGAAM makes use of elitist selection mechanism. ${ }^{20-22}$ In this mechanism, $P_{s}$ percent of the strings in the current population goes into the next population without change, while $P_{c}$ percent of the strings in the new population are generated by crossover between the parent strings. $P_{s}$ and $P_{c}$ are initially designated values. Parent strings to which crossover is applied are chosen fitness proportionally. The probability of a string having offspring in the next generation is inversely proportional to ${ }^{17}$

where $f(i)$ is the fitness value of $i$-th string.

$$
F(i)=\frac{f(i)}{\sum_{i} f(i)},
$$

By means of crossover, subsets of elements between two parent strings are exchanged. If the subsets consist of adjacent elements, it is an "ordered combination" crossover, and in "uniform combination" crossover each element is randomly selected. ${ }^{23}$ EGAAM adopts "uniform combination" crossover, with a number of elements to be exchanged randomly generated at each crossover operation. ${ }^{17}$ After the number of elements to be exchanged is generated (which is greater than the specified minimal number of elements), random integers determining positions of elements to be exchanged are generated and elements on those positions are exchanged.

In the process of mutation the population diversity is maintained, which prevents quick convergence to a local minimum. If the mutation probability is too large, mutation no longer improves the performance of the algorithm, since it enables losses of genetic information which can cause poor convergence. ${ }^{24}$ EGAAM applies adaptive mutation concept ${ }^{17}$ to overcome the phenomenon that real-coded GA can be blocked from further progress. ${ }^{25,26}$ In adaptive mutation scheme, $P_{m}$ percent of completely new strings are introduced in every generation. The new strings are generated in the same way like that during initial population generation, but a solution may exists within narrowed boundaries to provide that more strings are generated in the area. The boundaries are narrowed according to equations ${ }^{17}$

$$
p_{\text {new }-u}(k)=p_{\text {old }-u}(k)-c\left(p_{\text {old }-u}(k)-\mu(k)\right),
$$




$$
p_{\text {new-l }}(k)=p_{\text {old }-l}(k)+c\left(\mu(k)-p_{\text {old }-l}(k)\right)
$$

where $\mu(k)$ is the average value of parameter $p(k)$ in the current population, and $c$ is a specified positive number $c<1$. To avoid excessive narrowing of the boundaries, lower boundary was limited to be less than maximal allowed lower boundary, and upper boundary was limited to be greater than minimal allowed upper boundary.

The objective function plays the role of a measure of the quality of the obtained solution. To achieve the desired reflectance dependence on the wavelength $\lambda$, we assume the following objective function

$$
F=\left\{\sum_{i=1}^{n_{p}}\left(R_{c}-R_{d}\right)^{2} \exp \left(-\frac{\left(\lambda-\lambda_{0}\right)^{2}}{2 \sigma^{2}}\right)\right\}+\Delta,
$$

where $n_{p}$ is the number of points in which we calculate the reflectance $R_{c}, R_{d}$ is the desired reflectance, and $\lambda_{0}$ is the wavelength where peak reflectivity should be achieved. $\Delta$ is the penalty function, which is set to zero if the composition difference constraints are satisfied, or set to a specified value $10 \leq \Delta \leq 100$ if the composition difference between adjacent layers is larger than the required value. The desired reflectance is equal to 0.8 in the range $\left[\lambda_{0}-\Gamma, \lambda_{0}+\Gamma\right]$ where $\Gamma$ is the half-width of reflectance peak, and equal to zero outside that range. The exponential factor in Eq. (5) provides enhancement of the peak at $\lambda_{0}$.

We shall briefly present the theoretical background of transmission of electromagnetic wave through a series of layers with the complex index of refraction $N_{i}=n_{i}-\mathrm{i} k_{i}$ and thickness $d_{i}$, which are placed between the incident and substrate media with the index of refraction values $n_{0}$ and $N_{s}$. The equations are given for the case of perpendicular polarization only, since the procedure for parallel polarization is completely analogous, Electric and magnetic fields of the incident $\left(E_{I}, H_{I}\right)$ and transmitted $\left(E_{I I}, H_{I I}\right)$ waves are related in a following manner ${ }^{27,28}$ for one dielectric layer

$$
\left[\begin{array}{c}
E_{I} \\
H_{I}
\end{array}\right]=M \cdot\left[\begin{array}{l}
E_{I I} \\
H_{I I}
\end{array}\right],
$$

where

$$
M=\left[\begin{array}{cc}
\cos \left(k_{0} h\right) & i \frac{1}{Y_{i}} \sin \left(k_{0} h\right) \\
i Y_{i} \sin \left(k_{0} h\right) & \cos \left(k_{0} h\right)
\end{array}\right] .
$$

Time dependence of the electric field has the form $\exp (i \omega t), k_{0}=2 \pi / \lambda$ is the wave number of the incident electromagnetic wave, $h=N_{i} \cdot d \cdot \cos \theta_{i}, \theta_{i}$ is the angle of the electric field within the layer, and $Y_{i}$ is given by

$$
Y_{i}=\sqrt{\frac{\varepsilon_{0}}{\mu_{0}}} N_{i} \cos \theta_{i}
$$

In the case that there are $L$ layers between the incident and substrate media, we can assign the matrix in the form of Eq. (7) to each layer. The connection between electric and magnetic fields before $\left(E_{I}, H_{I}\right)$ and after $\left(E_{L+1}, H_{L+1}\right)$ the multilayer structure is described by

$$
\left[\begin{array}{l}
E_{I} \\
H_{I}
\end{array}\right]=\left(\prod_{i=1}^{N} M_{i}\right) \cdot\left[\begin{array}{l}
E_{L+1} \\
H_{L+1}
\end{array}\right]=\left[\begin{array}{ll}
m_{11} & m_{12} \\
m_{21} & m_{22}
\end{array}\right] \cdot\left[\begin{array}{c}
E_{L+1} \\
H_{L+1}
\end{array}\right]
$$

Fresnel coefficients are then given with

$$
r=\frac{Y_{0} \cdot m_{11}+Y_{0} \cdot Y_{s} \cdot m_{12}-m_{21}-Y_{s} \cdot m_{22}}{Y_{0} \cdot m_{11}+Y_{0} \cdot Y_{s} \cdot m_{12}+m_{21}+Y_{s} \cdot m_{22}},
$$




$$
t=\frac{2 Y_{0}}{Y_{0} \cdot m_{11}+Y_{0} \cdot Y_{s} \cdot m_{12}+m_{21}+Y_{s} \cdot m_{22}},
$$

where $n_{0}$ and $N_{s}$ are the index of refraction values of the incident and substrate medium, respectively, while $Y_{0}$ and $Y_{s}$ are the corresponding admitance values defined in the same manner as the $Y_{i}$ in equation (8). Ratios of the intensities of the transmitted and incident waves and the reflected and incident waves are described by the transmittance $T=t t^{*}$ $\operatorname{Re}\left(N_{s} \cdot \cos \theta_{s}\right) /\left(n_{0} \cdot \cos \theta_{0}\right)$ and reflectance $R=r r^{*}$, where $r^{*}$ and $t^{*}$ are the complex conjugated values of the Fresnel coefficients $r$ and $t$. In the structure air/sapphire/ $\mathrm{Al}_{\mathrm{x}} \mathrm{Ga}_{1-\mathrm{x}} \mathrm{N}$ reflector/GaN, since we have an optically thick, transparent substrate, it is necessary to introduce the correction for the reflection from the sapphire-air surface. The reflectance and transmittance at sapphire-air surface in case of normal incidence are $R_{s}=\left(\left(n_{s}-1\right) /\left(n_{s}+1\right)\right)^{2}$ and $T_{s}=4 n_{s} /\left(n_{s}+1\right)^{2}$. The corrected values for reflectance and transmittance ${ }^{29}$ are expressed as:

$$
\begin{aligned}
& \bar{R}=R+\frac{T T^{\prime} R_{s}}{1-R^{\prime} R_{s}}, \\
& \bar{T}=\frac{T T_{s}}{1-R^{\prime} R_{s}},
\end{aligned}
$$

where $R^{\prime}$ and $T^{\prime}$ are reflectance and transmittance of the back side of a multi-layers.

\section{RESULTS AND DISCUSSION}

We have taken into account the dependence of the index of refraction on wavelength for all the materials which are taken into account, including the glass substrate which is assumed to be non-absorbing with the refractive index dependency in the form $n=A+B / \lambda^{2}$. The index of refraction data for copper-phthalocyanine and N,N'-dimethylperylene-3,4:9,10dicarboximide have been modeled using a modified oscillator model. ${ }^{18}$ The index of refraction of ITO was calculated using the model given in Ref. ${ }^{19}$ The experimental data for TPD, ${ }^{20}$ which is transparent in the region of interest, have been modeled using the same expression as for the glass substrate.

Due to small differences in the refractive index of the organic materials considered, method a) does not produce good results not even for very large number of layers. Therefore, we will show here the results of asymmetric designs, procedures $b$ ) and c). Figure 2 shows the reflectivity of GA optimized filters using methods b) and c) for 30 and 40 layers. It can be observed that the method c) obtains higher value of the reflectance peak compared to method $b$ ). This is to be expected due to greater flexibility of the design method c). Figure 3 shows the calculated reflectivity of GA optimized organic Bragg reflectors obtained by method c) for different number of layers. It can be observed that in all cases obtained reflectivity is below $80 \%$. This may still be sufficient for microcavity OLEDs applications. However, better results would be obtained if the index of refraction data were avaialable for more organic materials which are transparent in the spectral region 400-600 nm. Doped polymer layers may offer a solution to the problem of small refractive index differences and hence low reflectivity even for a large number of layers. However, since evaporation usually offers better thickness control than spin-coating technique, reflective filters consisting of evaporated dye layers may be preferrable.

Most of organic materials have very small refractive index differences in the transparent spectral region, which raises the required number of layers needed to achieve higher reflectance. The conventional quarter-wave reflectors using two dielectrics with high difference of refractive indices require significantly fewer number of layers. However, disadvantages of using dielectric Bragg mirrors are: 1) ITO electrode must be placed inside the cavity thus increasing the effective cavity length and reducing quality factor 2) ITO needs to be sputtered on top of the dielectric mirror, and ITO layer quality may significantly differ from sample to sample since the sputtering of this material is very sensitive to a number of factors. If organic Bragg reflectors are used, cavity may be shorter, commercial ITO coated glass substrates can be used, but the fabrication of an asymmetric Bragg mirror consisting of large number of layers may prove to be difficult. Figure 4 shows the reflectance of a filter design using method $b$ ) for dielectric materials for different number of layers. The index of refraction of the considered dielectric materials $\left(\mathrm{SiO}, \mathrm{SiO}_{2}\right.$, and $\left.\mathrm{Si}_{3} \mathrm{~N}_{4}\right)$ has been modeled using a model described in Ref. ${ }^{21}$ It can be observed that the height of the reflectance peak is larger for the reflector made of dielectric materials compared to an all-organic reflector, as well as the half-width of the peak. This is due to larger difference in the refractive index of the available materials. because of the reasons specified above, i.e. possibility of making shorter cavities and use of 
commercially available ITO coated glass substrates, organic reflectors may still be promising for improving the performance of microcavity OLEDs.

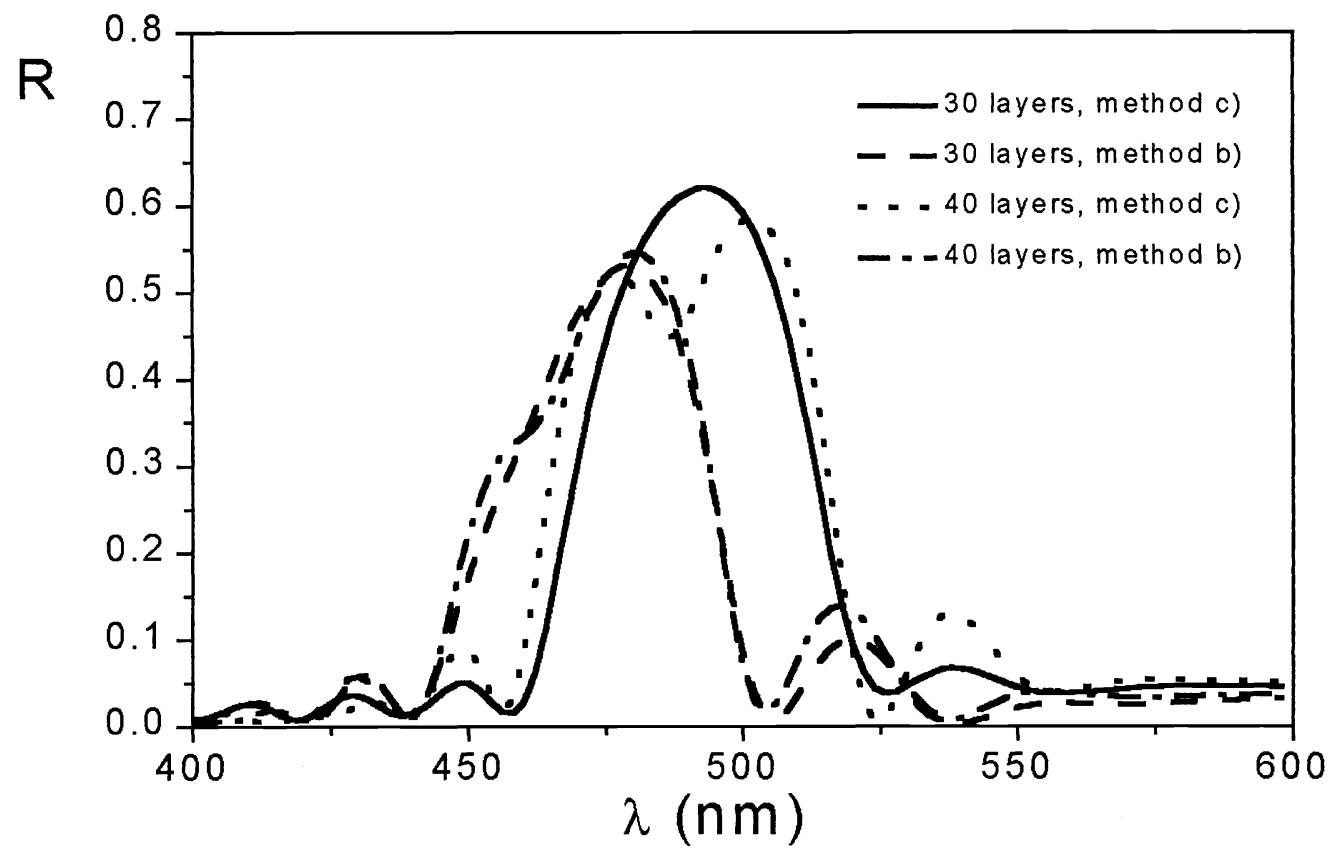

Fig. 2 Reflectance of filters for design type b) and c).

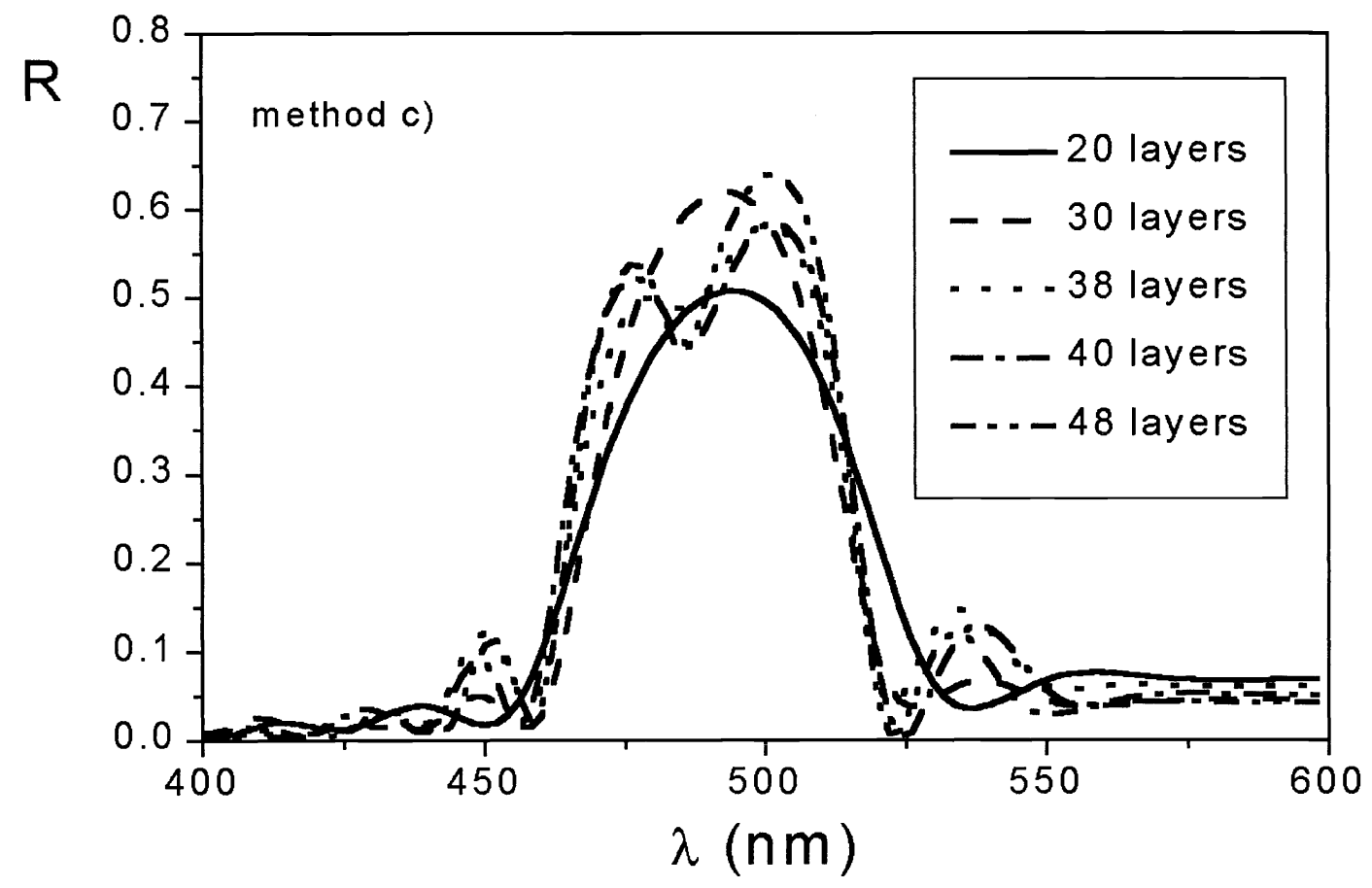

Fig. 3 Reflectance of filters designed with method c) for different number of layers. 


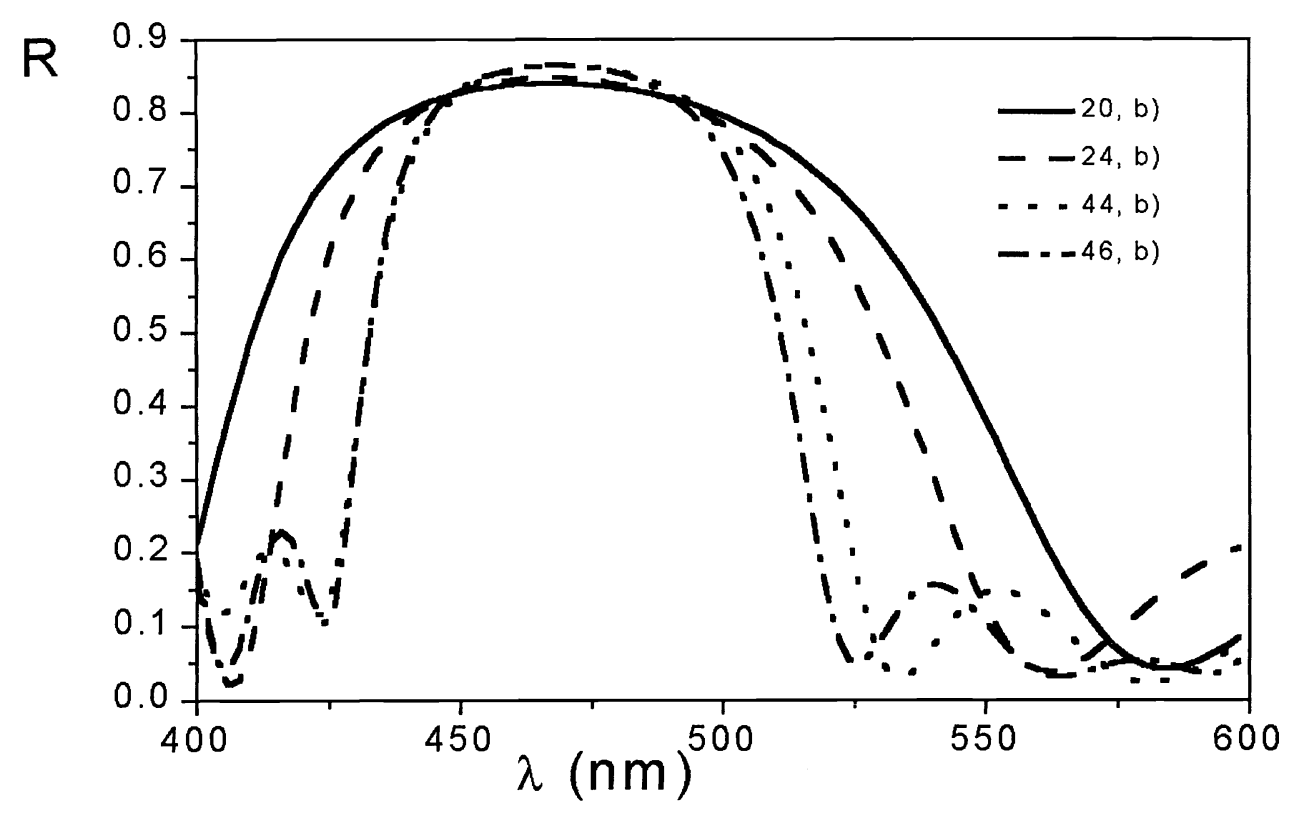

Fig. 4 Reflectance of filters designed with method b) for dielectric materials.

\section{CONCLUSION}

We have presented a genetic algorithm based method for the design of the reflective filters consisting of organic materials. Such reflective filters may have potential use in microcavity based OLEDs. Since the layers are organic semiconductors, the hole injecting anode (usually ITO) could be placed outside the cavity thus improving the quality factor. However, further investigation is needed concerning the transport processes in such a device. Nevertheless, the described design method represents a useful tool in filters design since it is highly versatile, i.e. it enables easy changes of design objective by simple manipulations of the objective function, and the materials involved can be changed by using a list of different materials from which layers are chosen based on the defined material code.

\section{ACKNOWLEDGEMENTS}

The authors would like to thank the RGC earmarked grant of Hong Kong and the University of Hong Kong CRCG research grant for financial support.

\section{REFERENCES}

1. K. Neyts, P. De Visschere, D. K. Fork, and G. B. Anderson, "Semitransparent metal or distributed Bragg reflector for wide-viewing-angle organic light-emitting-diode microcavities", J. Opt. Soc. Am. B, vol. 17, pp. 114-119, Jan. 2000.

2. P. K. H. Ho, D. S. Thomas, R. H. Friend, and N. Tessler, "All-polymer optoelectronic devices", Science, vol. 285 (5425), pp. 233-236, July 1999.

3. T. Tsutsui, N. Takada, S. Saito, and E. Ogino, "Sharply directed emission in organic luminescent diodes with an opticalmicrocavity structure", Appl. Phys. Lett., vol. 65, pp. 1868-1870, Oct. 1994.

4. R. H. Jordan, L. J. Rothberg, A. Dodabalapur, and R. E. Slusher, "Efficiency enhancement of microcavity organic light emitting diodes", Appl. Phys. Lett., vol. 69, pp. 1997-1999, Sept. 1996

5. A. B. Djurišić, "Elite genetic algorithms with adaptive mutations for solving continuous optimization problems application to modeling of the optical constants of solids", Opt. Comm. 151, 147-159, 1998.

6. K. P. Wong and Y. W. Wong, "Floating-point number coding method for genetic algorithms", in Proc. ANZIIS-93, pp. 512-516, Perth, Western Australia, 1993. 
7. K. P. Wong and Y. W. Wong, "Genetic and genetic/simu;ated annealing approaches to economic dispatch", IEE Proc. Gen. Transm. Distrib. 141, pp. 507-513, 1994.

8. R. Vemuri and R. Vemuri, "Genetic algorithm for MCM partitioning", Elec. Lett. 30, pp. 1270-1272, 1994.

9. S. H. Clearwater and T. Hogg, "Problem structure heuristics and scaling behavior for genetic algorithms", Artificial intelligence 81, pp. 327-347, 1996.

10. R. R. Brooks, S. S. Iyengar and J. Chen, "Automatic correlation and calibration of noisy sensor readings using elite genetic algorithms", Artificial intelligence 84, pp. 339-354, 1996.

11. F. Curatelli, "Implementation and evaluation of genetic algorithms for system partitioning", Int. J. Electronics 78, pp. 435-447, 1995.

12. D. Raynolds and J. Gomatam, "Stochastic modeling of genetic algorithms", Artificial intelligence 82, pp. 303-330, 1996.

13. A. B. Djurišić, J. M. Elazar and A. D. Rakić,"Modeling the optical constants of solids using genetic algorithms with parameter space size adjustment", Opt. Comm. 134, pp. 407-414, 1997.

14. D. E. Goldberg, "Real-coded genetic algorithms, virtual alphabets, and blocking", Complex Systems 5, pp. 139-167, 1991.

15. M. Born and E. Wolf, Principles of Optics, Pergamon Press, New York, 1964.

16. E. Hecht, Optics, Addison-Wesley, New York, 1990.

17. B. Harbecke, "Coherent and incoherent reflection and transmission of multilayer structures", Appl. Phys. B 39, pp. 165-170, 1986.

18. A. B. Djurišić, T. Fritz and K. Leo, "Modeling the optical constants of organic thin films: impact of the choice of objective function", accepted for publication in J. Opt. A: Pure Appl. Opt. 2000.

19. S. Laux, N. Kaiser, A.Zöler, R. Götzelmann, H. Lauth, and H. Bernitzki, "Room-temperature deposition of indium tin oxide thin films with plasma ion-assisted evaporation", Thin Solid Films 335, pp. 1-5, 1998.

20. F. G. Celii, T. B. Harton, and O. F. Phillips, "Characterization of Organic thin films for OLEDs using spectroscopic ellipsometry”, J. Electron. Mater. 26, pp. 366-371, 1997.

21. A. B. Djurišić and E. H. Li, "Modeling the Refractive Index of Insulating Solids by Modified Lorentz Oscillator Model", Appl. Opt. 37, pp. 5291-5297, 1998. 